\title{
High risk and rapid appearance of multidrug resistance during tuberculosis treatment in Moldova
}

\author{
Helen E. Jenkins ${ }^{1,2}$, Valeriu Crudu ${ }^{3}$, Viorel Soltan ${ }^{3}$, Ana Ciobanu ${ }^{4}$, \\ Liliana Domente ${ }^{4}$ and Ted Cohen ${ }^{1,5}$
}

Affiliations: ${ }^{1}$ Brigham and Women's Hospital, Boston, MA, ${ }^{2}$ Harvard Medical School, Boston, MA, and ${ }^{5} \mathrm{Harvard}$ School of Public Health, Boston, MA, USA. ${ }^{3}$ Center for Health Policies and Studies, Chisinau, and ${ }^{4}$ Phthisiopneumology Institute, Chisinau, Moldova.

Correspondence: H.E. Jenkins, Brigham and Women's Hospital, 641 Huntington Avenue, Boston, MA 02115 , USA. E-mail: jenkins.helendagmail.com

ABSTRACT Multidrug-resistant tuberculosis (MDR-TB) is a serious problem in the former Soviet Union and may appear during TB treatment. We aimed to estimate the prevalence of, timing of and factors associated with MDR-TB diagnosis during TB treatment in Moldova, which was part of the former Soviet Union.

We analysed data on 3754 confirmed non-MDR-TB cases (between January 1, 2007 and December 31, 2010) in the Moldovan TB surveillance database, where patients provided sputum specimens for drugsusceptibility testing, multiple times, during treatment. We estimated the percentage of individuals with confirmed baseline non-MDR-TB that were diagnosed with MDR-TB during treatment, documented the time at which MDR-TB was diagnosed, and used a failure-time model to identify factors associated with MDR-TB diagnosis.

Between $7.2 \%$ and $9.2 \%$ of initially non-MDR-TB cases were diagnosed with MDR-TB during treatment. Half of these MDR-TB diagnoses occurred with 3 months of the initial diagnosis. An increased MDR-TB risk during treatment was associated with baseline resistance to first-line TB drugs (linear increase in risk per additional drug), previous incarceration and HIV co-infection.

MDR can appear rapidly during TB treatment. Policy considerations should emphasise management during early treatment by increasing ambulatory TB treatment to prevent nosocomial transmission, and ensuring universal rapid diagnostics access to prevent acquisition and transmission of drug resistance.

@ERSpublications

There is a high risk of multidrug resistance early on during tuberculosis treatment in Moldova http://ow.ly/tlqKZ

Earn CME accreditation by answering questions about this article. You will find these at the back of the printed copy of this issue or online at www.erj.ersjournals.com/site/misc/cmeinfo.xhtml

This article has supplementary material available from www.erj.ersjournals.com

Received: Nov 202013 | Accepted after revision: Dec 312013 | First published online: Feb 202014

Support statement: This work was supported by the National Institute of General Medical Sciences (grants U54GM088558 and DP2OD006663) and the National Institute of Allergy and Infectious Diseases at the National Institutes of Health (grant K01AI102944). The content is solely the responsibility of the authors and does not necessarily represent the official views of the National Institute of General Medical Sciences, the National Institute of Allergy and Infectious Diseases or the Office of the Director, National Institutes of Health. The funding source had no role in the study design, collection, analysis, or interpretation of the data, writing of the report or the decision to submit the paper for publication.

Conflict of interest: None declared.

Copyright @ERS 2014 


\section{Introduction}

Despite recent declines in the global estimated incidence of and mortality due to tuberculosis (TB) [1], the highest ever levels of multidrug-resistant (MDR) TB, i.e. TB resistant to at least isoniazid and rifampicin, were reported in 2012 [2,3]. MDR-TB control is often hindered by challenges in detecting resistant disease because of limited access to both drug-susceptibility testing (DST) and quality-assured treatment for MDR-TB [4]. In 2012, of the 450000 estimated MDR-TB cases among notified pulmonary TB cases globally, only $17 \%$ were diagnosed and initiated on the appropriate treatment [1].

Countries of the former Soviet Union (FSU) have reported proportions of TB cases with MDR-TB several times higher than those detected elsewhere $[1,2,5]$. Moldova, with a population of four million [6], was part of the FSU and has, like many other FSU countries, a high reported percentage of TB cases with MDR-TB ( $24 \%$ of treatment-naïve cases and $62 \%$ of previously treated cases [7]) and similar policies. However, unlike many other high-burden countries, Moldova has made substantial investments to address the MDR-TB crisis $[5,8]$. In particular, current national policy mandates DST for all culture-positive cases at initial diagnosis and several additional times during treatment, an exceptionally rare testing policy in high TB-prevalence countries.

Despite using internationally recommended first-line treatment regimens, only $68 \%$ of patients diagnosed with non-MDR-TB at baseline between January 1, 2007 and December 31, 2010 in Moldova were cured or completed treatment [9]. One factor limiting successful therapy is the appearance of MDR-TB during treatment for non-MDR-TB, which may occur by one of three mechanisms: 1) sporadic drug-resistant mutants are selected during therapy due to functional monotherapy (i.e. "acquired" drug resistance) [10], 2) a patient is re-infected with an MDR-TB strain during treatment [11, 12], or 3) a patient had a mixedstrain infection in which first-line treatment unmasked a MDR-TB strain that was present at baseline but undetected [13-15]. Here, we use "appearance" to mean MDR-TB during treatment resulting from any of these mechanisms, and "acquisition" to refer only to mechanism 1. In Moldova and other parts of the FSU, the appearance of MDR-TB during treatment for non-MDR-TB may be contributing to low rates of successful outcomes (typically observed among MDR-TB patients [16]) and to the high population-level burden of MDR-TB. Here, we use the Moldovan TB-surveillance data to address several questions: 1) How frequently are TB patients diagnosed with MDR-TB during treatment? 2) When does this occur? 3) Which patient characteristics are associated with MDR-TB appearance during treatment? We also discuss the implications of our results for TB policies in the FSU.

\section{Methods}

Study setting

In Moldova, TB cases are diagnosed by sputum-smear microscopy, culture, and/or abnormal radiography in the presence of symptoms. During the study period (between January 1, 2007 and December 31, 2010), 92\% of TB cases received culture testing and $94 \%$ of culture-positive cases received DST [7]. Culture and DST are performed at four laboratories, which have all passed external quality assurance from the SupraNational Reference Laboratory Forschungszentrum Borstel, Borstel, Germany [17]. DST was done on solid culture using the absolute concentration method throughout the study period; antibiotic concentrations used were $1 \mu \mathrm{g} \cdot \mathrm{mL}^{-1}$ for isoniazid, $40 \mu \mathrm{g} \cdot \mathrm{mL}^{-1}$ for rifampicin, $2 \mu \mathrm{g} \cdot \mathrm{mL}^{-1}$ for ethambutol and $5 \mu \mathrm{g} \cdot \mathrm{mL}^{-1}$ for streptomycin [18]. The mycobacteria growth indicator tube BACTEC MGIT 960 (Becton, Dickinson and Company, Franklin Lakes, NJ, USA; a liquid culture and DST system) was phased in during the study period and was being used for all TB cases by 2009. The average time to DST results was 6-7 weeks and 3-4 weeks for solid-culture methods and MGIT 960, respectively. National policy states that all TB patients without MDR-TB that begin treatment provide sputum samples at four time points; 2-3 months, 3-4 months and 5 months from the beginning of treatment and at treatment completion. All samples receive microscopy and culture and, if culture positive, receive DST. We defined follow-up tests as those carried out at time points after the initial testing and diagnosis.

In Moldova, TB treatment follows the World Health Organization directly observed treatment, short-course (DOTS) strategy (online supplementary material) [19, 20]. In contrast with WHO recommendations [21], but as in many FSU countries, Moldovan policy is to hospitalise TB cases for the intensive treatment phase (average length of stay is 92 days [22]). The remaining treatment is received via ambulatory care. Supply of quality-assured first-line drugs is guaranteed for all TB patients [22]. However, supply of second-line drugs is limited and new patients (those with $<1$ month of previous $\mathrm{TB}$ treatment) and previously treated patients (those with at least 1 month of previous TB treatment) without a history of default are prioritised. Most TB patients in Moldova are hospitalised and begin treatment on the day of diagnosis.

While hospitalised, MDR-TB patients are separated from those without MDR-TB (in separate buildings or on separate floors within the same building). However, among those without MDR-TB, those with resistance to some first-line drugs are not separated from those with pan-susceptible TB. Before DST results 
are available, all TB cases start receiving the WHO recommended regimen for drug-susceptible TB and MDR-TB cases are only separated after DST confirmation.

\section{Data source}

We analysed routinely collected surveillance data of all TB cases reported in Moldova between January 1, 2007 and December 31, 2010. We focused on individuals that were confirmed, through DST, not to have MDR-TB (non-MDR-TB). All laboratory results and treatment outcomes are recorded in an online database along with demographic data collected at initial diagnosis. The National Tuberculosis Programme and the National Centre of Health Management verify all data and if there are inconsistencies, compare the online data with paper records at the TB facilities. We defined an MDR-TB diagnosis as a confirmation through DST of resistance to at least isoniazid and rifampicin, and non-MDR-TB cases are those with confirmed susceptibility to isoniazid and/or rifampicin. TB cases diagnosed in the penitentiary system were excluded from our study since follow-up test results from these patients into the database were inconsistently reported.

This study used non-identifiable, clinical data collected during routine care and, therefore, was deemed exempt by the Partners Institutional Review Board, Boston MA, USA. This study was approved by the Research Ethics Committee of the Phthisiopneumology Institute in Moldova.

\section{Statistical analysis}

Percentage with and timing of MDR-TB diagnosis during treatment for non-MDR-TB

We estimated the percentage of patients with non-MDR-TB at baseline that later had an MDR-TB diagnosis confirmed through DST in that treatment episode. There were several criteria for exclusion from our study (fig. 1 and online supplementary material).

We investigated the timing of MDR-TB diagnosis and of first follow-up sputum collection for all cases with censored or MDR-TB diagnosis times in the first year after initial diagnosis.

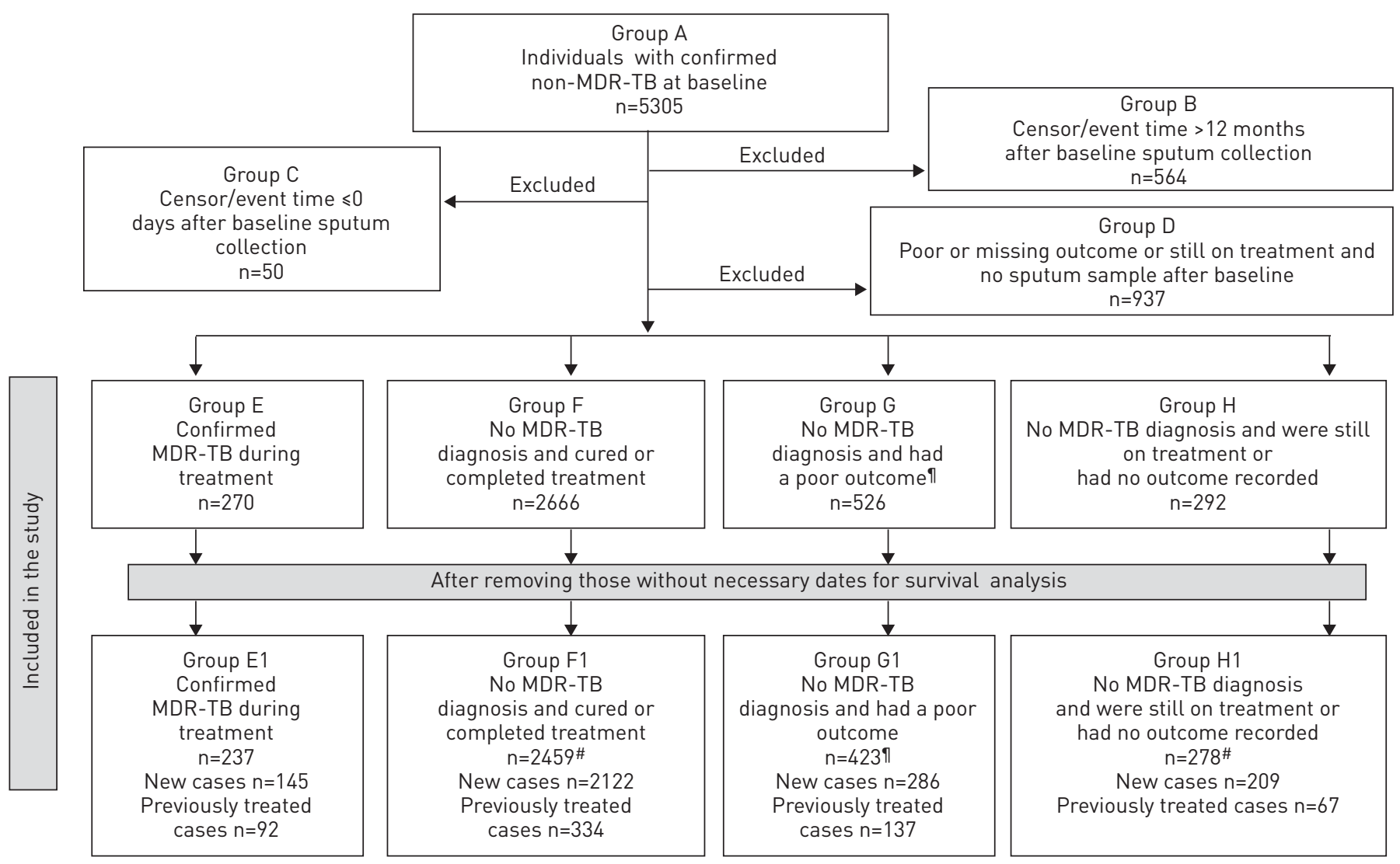

FIGURE 1 Flow chart of inclusions and exclusions for the study. All outcomes (or lack of outcome) are as recorded in the Moldovan national tuberculosis (TB) database as of July 2011. MDR: multidrug resistant. " : the total number includes patients whom initiated treatment abroad and, therefore, are not in either new or previously treated categories; ": poor outcome includes died, defaulted on treatment and failed treatment. 
Risk factors for MDR-TB diagnosis during treatment for non-MDR-TB

We used two failure-time models for interval censored data (one for new TB cases and one for previously treated TB cases) to identify risk factors for MDR-TB diagnosis during treatment (online supplementary material) $[23,24]$. Cases were those that had a confirmed MDR-TB diagnosis through DST at some point in their treatment episode (chronologically later than the baseline non-MDR-TB diagnosis). Controls were those who were confirmed non-MDR-TB at baseline and did not have confirmation of MDR-TB at any point in that treatment episode. Controls were censored either 1) at treatment outcome date if their outcome was cured or completed treatment or 2) for those that went on to have an unsuccessful outcome, at the point when a sputum sample was collected that was either negative for TB or confirmed through DST to be non-MDR. All analyses were carried out in SAS, version 9.2 (SAS Institute, Inc., Cary, NC, USA) and we used the PROC LIFEREG statement for the failure-time models [25].

\section{Results \\ Percentage of non-MDR-TB cases that had MDR-TB diagnosed during treatment}

During the study period, 5305 TB cases had confirmed non-MDR-TB at baseline. Of these, 1551 (29\%) were excluded from our study (groups B, C and D in fig. 1 and online supplementary material). Of the remaining 3754 cases (i.e. combination of groups E, F, G and H in fig. 1), 270 (7.2\%) had an MDR-TB diagnosis during treatment $(5.3 \%$ of new cases and $14.9 \%$ of previously treated cases). Since some patients in groups $\mathrm{G}$ and $\mathrm{H}$ may have had a poor outcome or still be on treatment due to undiagnosed MDR-TB infection, this approach may under-estimate the true percentage of cases in whom MDR-TB appeared. If we exclude groups $\mathrm{G}$ and $\mathrm{H}$ from the denominator and only include those in whom MDR-TB definitely did or did not appear (i.e. groups E and F in fig. 1), 270 (9.2\%) out of 2936 were diagnosed with MDR-TB (6.5\% of new cases and $22.6 \%$ of previously treated cases. This approach may over-estimate the true percentage that were diagnosed with MDR-TB and, therefore, we conclude that at least $7.2 \%$ but no more than $9.2 \%$ of nonMDR-TB cases were diagnosed with MDR-TB during the first year of treatment. Baseline patient characteristics are provided in the online supplementary material.

\section{Timing of follow-up sputum collection and MDR-TB diagnosis}

The majority of events in which initially non-MDR-TB patients had a subsequent MDR-TB diagnosis happened at the first follow-up opportunity $(72 \%$ and $80 \%$ of new and previously treated cases, respectively) (table 1). Of all cases diagnosed with MDR-TB within 1 year of initial non-MDR-TB diagnosis, half were diagnosed with MDR-TB within or soon after the first 3 months (fig. 2a). Of all cases diagnosed with MDR-TB during the first year of treatment, $70 \%$ of new cases and $46 \%$ of previously treated cases had sputum taken for the first time since baseline testing by 90 days after initial diagnosis (fig. 2b).

\section{Individual-level risk factors for MDR-TB diagnosis during treatment for non-MDR-TB}

In a multivariable analysis, we identified several statistically significant risk factors associated with MDR-TB diagnosis during non-MDR-TB treatment (table 2). In particular, among new cases, groups that had an increased risk of MDR-TB diagnosis during treatment included those that had previously been in detention, TB cases that lived alone, those with a higher degree of lung pathology, people with concurrent HIV infection, younger age and cases with baseline resistance to drugs. Table 3 demonstrates the relationship between baseline resistance and subsequent diagnosis of MDR-TB during treatment. Of treatment-naive TB cases with no baseline

TABLE 1 The percentage of cases that are diagnosed with multidrug-resistant tuberculosis (MDR-TB) during treatment for non-MDR-TB by sputum collection time

Sputum collection number after initial diagnosis

\begin{tabular}{cccc}
\hline $\mathbf{1}$ & $\mathbf{2}$ & $\mathbf{3}$ & $\mathbf{4}$ \\
$104(72)$ & $34(23)$ & $6(4)$ & $1(1)$ \\
$74(80)$ & $16(17)$ & $2(2)$ & $0(0)$ \\
$68(41,354)$ & $126(56,340)$ & $157(119,178)$ & $153(153,153)$ \\
$92(16,340)$ & $153.5(59,355)$ & $183(122,244)$ & Not applicable
\end{tabular}

Data are presented as $\mathrm{n}(\%)$ or $\mathrm{n}$ (minimum, maximum). DST: drug susceptibility testing. 
a)

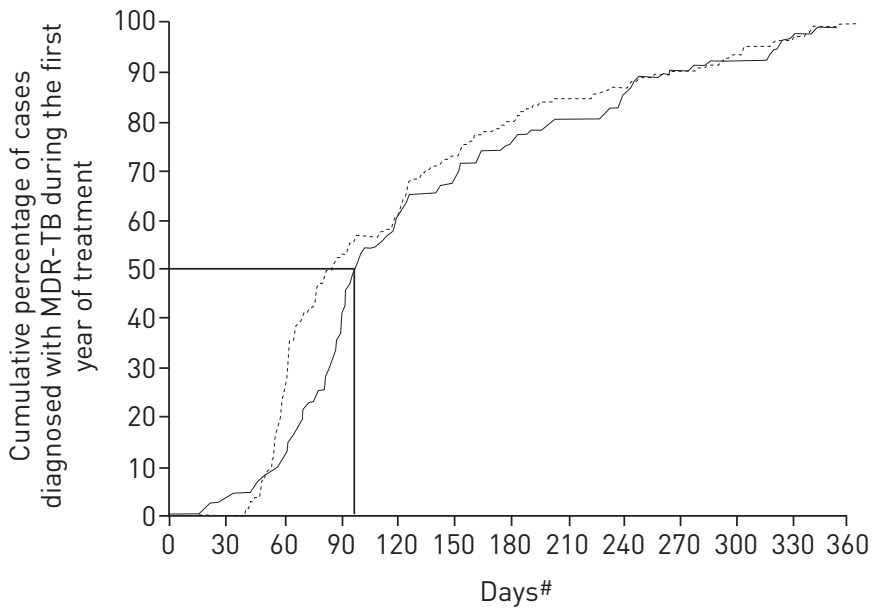

b)

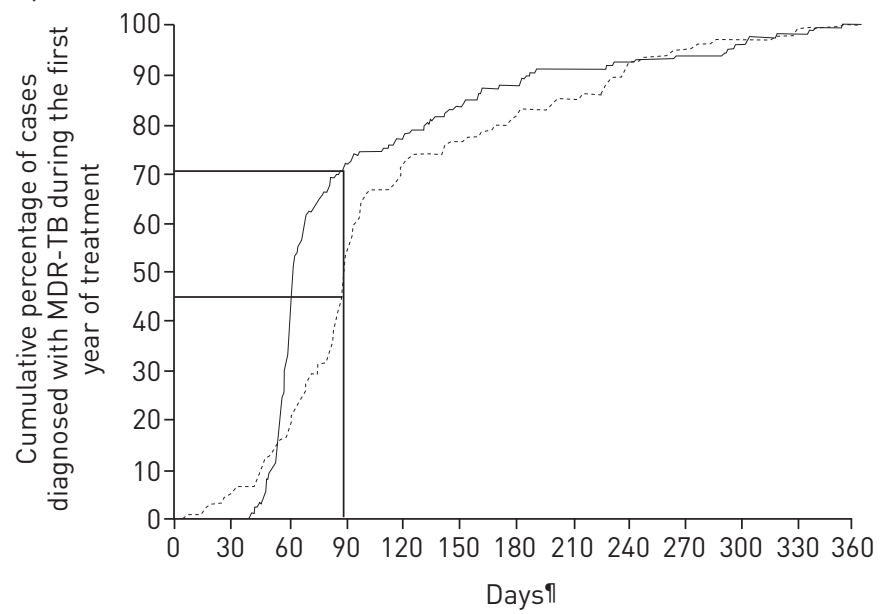

FIGURE 2 Cumulative percentage of non-multidrug-resistant tuberculosis (non-MDR-TB) cases in whom MDR-TB appeared ( $\mathrm{n}=237$ ) during the first year of treatment by a) time after initial diagnosis at which MDR-TB is detected, and b) time after initial diagnosis at which the first follow-up sputum collection occurred. Data are shown separately for new cases (solid line, $\mathrm{n}=145$ ) and previously treated cases (dotted line, $\mathrm{n}=92$ ). Straight lines indicate in a) the days after initial diagnosis at which $50 \%$ of cases had been diagnosed with MDR-TB (87 days for new cases and 99 days for previously treated cases), and in b) the percentage of cases where sputum had been collected for follow-up testing by 90 days after initial diagnosis $(70 \%$ of new cases and $46 \%$ of previously treated cases). ": The number of days after initial TB diagnosis that MDR-TB diagnosis was made; ${ }^{\circ}$ : the number of days after initial TB diagnosis that first follow-up sputum collection occurred.

resistance, only 3.6\% were diagnosed with MDR-TB during treatment. Conversely, one-third of treatment-naïve cases with resistance to three first-line drugs were subsequently diagnosed with MDR-TB.

\section{Discussion}

In this study, we found that $\sim 75 \%$ of MDR-TB diagnoses were made at the first follow-up test after initial diagnosis (fig. $2 \mathrm{~b}$ shows the distribution of first follow-up sputum collection from which the diagnosis was made). While we could not assess the precise timing of the appearance of MDR, the fact that $50 \%$ of all diagnoses of MDR-TB occurred within 3 months of TB diagnosis and treatment initiation, suggests that the majority of MDR-TB cases appeared in the earliest weeks of treatment or were already present at baseline but undetected.

We found that between 7.2\% and 9.2\% of non-MDR-TB cases in Moldova (between January 1, 2007 and December 31, 2010) were subsequently diagnosed with MDR-TB during treatment. This is consistent or slightly higher than what was found in Tomsk, Russia (7.3\%) [26]. However, even our upper bound of 9.2\% may underestimate the true prevalence of MDR-TB appearance during TB treatment, since nearly 1000 cases $(18 \%$ of total) initially diagnosed with non-MDR-TB had a poor or missing outcome and no followup sputum collection during treatment and excluded from our study. It is possible that MDR-TB was more likely to appear in these patients than those patients included in our study, due to their poor outcomes.

Due to the resources necessary for culture and DST, few high TB-incidence countries routinely perform culture and DST at baseline and during TB treatment, as recommended by WHO [27]. The relatively large sample size and presence of externally quality-assured DST to rule out MDR-TB at baseline are major strengths of this study. It should be noted that the quality of DST is consistent regardless of whether the DST was carried out on the baseline sample or subsequent samples. We found that a higher degree of lung pathology was associated with MDR-TB appearance, consistent with a study examining the appearance of extensively drug-resistant TB in MDR-TB patients [28] in which the authors suggested that this may be due to the greater bacillary load within cavitary lesions, thereby increasing the probability of mutations associated with drug resistance. A higher degree of lung pathology, HIV infection and baseline resistance were also associated with MDR-TB during treatment in a study in California, USA [29] and HIV coinfection was a risk factor for acquired resistance to second-line drugs in another study in the USA [30]. Previous incarceration, younger age and HIV co-infection were also associated with MDR-TB at initial diagnosis in this population [7].

We found that being infected with TB resistant to first-line drugs was associated with MDR-TB diagnosis during treatment. This is consistent with a higher risk of acquired drug resistance during treatment, possibly through functional monotherapy [31] and is consistent with other studies in the FSU [32, 33]. This could have occurred between initial diagnosis and availability of DST results if cases infected with TB that was 
TABLE 2 Individual-level risk factors for multidrug-resistant tuberculosis (MDR-TB) diagnosis during treatment among cases without MDR-TB at baseline in Moldova"

Variable

\begin{tabular}{cc}
\hline Unadjusted HR & Adjusted HR \\
$(95 \% \mathrm{Cl})$ & $(95 \% \mathrm{Cl})$
\end{tabular}

$(95 \% \mathrm{Cl})$

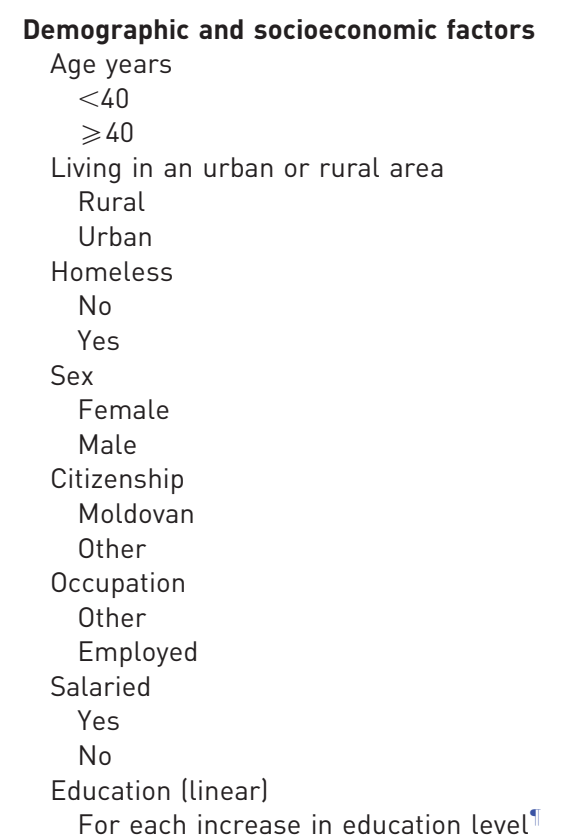

Spent $>3$ months outside Moldova during previous 12 months

No

Yes

Was previously in detention No

Yes

Household size

Living with others

Living alone

Number of children in the household At least one None

Lives with someone with diagnosed TB No

Yes

Region of residence

HIV status and TB-related factors

Degree of lung pathology

Infiltration

Destruction

Smear microscopy result Negative/untested/result unknown Positive

Culture positive, linear graded 1-3

For each increase in grade

HIV status

Negative/untested/result unknown

Positive

Presence of resistance to any first-line drugs at baseline $^{+}$

None

Any drug resistance present

New TB cases

Reference 0.34 (0.15-0.79)

$0.26(0.11-0.60)$

Reference

$1.89(0.86-4.12)$

Reference

$2.58(0.43-15.65)$

Reference

$1.55(0.60-3.98)$

Reference

$2.86(0.03-299.2)$

Reference

$0.22(0.07-0.73)$

$0.28(0.05-1.40)$

Reference

$2.97(1.13-7.81)$

$0.87(0.52-1.46)$

$1.48(0.39-5.59)$

Reference

3.67 (1.54-8.79)

Reference

11.97 (3.59-39.91)

Reference

$4.85(1.92-12.22)$

Reference

2.22 (0.95-5.19)

Reference

2.10 (0.52-8.52)

Could not be estimated

$$
\begin{aligned}
& \text { Reference } \\
& 2.40(1.01-5.71)
\end{aligned}
$$

Reference

2.10 (0.87-5.08)

$1.47(0.91-2.38)$

Reference

12.96 (3.16-53.15)

Reference $14.31(5.69-35.96)$
Previously treated TB cases

Unadjusted HR Adjusted HR

(95\% CI)

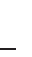




\section{TABLE 2 Continued}

Variable
New TB cases

\begin{tabular}{cc}
\hline $\begin{array}{c}\text { Unadjusted HR } \\
(95 \% \mathrm{Cl})\end{array}$ & $\begin{array}{c}\text { Adjusted HR } \\
(95 \% \mathrm{Cl})\end{array}$
\end{tabular}

Previously treated TB cases

\begin{tabular}{cc}
\hline $\begin{array}{c}\text { Unadjusted HR } \\
(\mathbf{9 5 \%} \mathbf{~ C l})\end{array}$ & $\begin{array}{c}\text { Adjusted HR } \\
(\mathbf{9 5 \%} \mathbf{C l})\end{array}$ \\
$4.48(2.56-7.85)$ & $2.85(1.51-5.36)$ \\
Reference & \\
$4.02(1.35-11.92)$ & \\
Reference & Reference \\
$111.16(21.72-569.0)$ & $20.50(4.83-86.94)$ \\
& \\
Reference & Reference \\
49.58 (10.85-226.6) & $2.54(0.47-13.76)$ \\
Reference & \\
6.44 (2.24-18.49) &
\end{tabular}

Unadjusted and adjusted hazard ratios (HR) and $95 \%$ confidence intervals from univariable and multivariable models are presented for both new and previously treated cases. Cells left blank indicate that variable was not included in the multivariable model. \#: between January 1, 2007 and December 31, 2010; ${ }^{\top}$ : categories in increasing order: no education, primary, secondary, specialised secondary, higher; ${ }^{+}$: all of these variables were potentially highly correlated (online supplementary material).

TABLE 3 Frequency of each potential baseline tuberculosis (TB) drug resistance profile among non-multidrug-resistant (nonMDR) TB cases in Moldova" and the number of those that were diagnosed with MDR-TB during treatment

\section{Baseline resistance profile}

New cases

\begin{tabular}{cc}
\hline $\begin{array}{c}\text { With each resistance } \\
\text { profile }\end{array}$ & $\begin{array}{c}\text { Diagnosed with MDR-TB } \\
\text { during treatment }\end{array}$
\end{tabular}

Previously treated cases

\begin{tabular}{|c|c|c|c|c|}
\hline & & \\
\hline & $\begin{array}{l}\text { With each resistance } \\
\text { profile }\end{array}$ & $\begin{array}{l}\text { Diagnosed with MDR-TB } \\
\text { during treatment }\end{array}$ & $\begin{array}{l}\text { With each resistance } \\
\text { profile }\end{array}$ & $\begin{array}{l}\text { Diagnosed with MDR-TB } \\
\text { during treatment }\end{array}$ \\
\hline None detected & 2114 & $76(3.6)$ & 413 & $46(11.1)$ \\
\hline R-mono & 26 & $2(7.7)$ & 12 & $6(50.0)$ \\
\hline E-mono & 25 & $3(12.0)$ & 3 & $0(0.0)$ \\
\hline $\mathrm{H}+\mathrm{S}$ & 149 & $17(11.4)$ & 68 & $17(25.0)$ \\
\hline $\mathrm{R}+\mathrm{E}$ & 2 & $0(0.0)$ & 3 & 1 (33.3) \\
\hline $\mathbf{R}+\mathbf{S}$ & 10 & $1(10.0)$ & 6 & 5 (83.3) \\
\hline$E+S$ & 6 & $1(16.7)$ & 2 & $0(0.0)$ \\
\hline $\mathrm{H}+\mathrm{E}+\mathrm{S}$ & 50 & $15(30.0)$ & 15 & $7(46.7)$ \\
\hline Any $\mathrm{E}$ & 101 & 25 (24.8) & 30 & $15(50.0)$ \\
\hline Any $S$ & 472 & $51(10.8)$ & 153 & 36 (23.5) \\
\hline Any resistance & 648 & $69(10.6)$ & 213 & $46(21.6)$ \\
\hline Any 1 drug & 408 & $29(7.1)$ & 112 & $9(8.0)$ \\
\hline Any 2 drugs & 178 & $21(11.8)$ & 81 & 25 (30.9) \\
\hline Any 3 drugs & 57 & 19 (33.3) & 19 & $11(57.9)$ \\
\hline Total & 2762 & 145 (5.2) & 626 & 92 (14.7) \\
\hline
\end{tabular}

Data are presented as $\mathrm{n}$ or $\mathrm{n}(\%)$. $\mathrm{H}$ : isoniazid; R: rifampicin; E: ethambutol; S: streptomycin. Only 43 cases were tested for resistance to pyrazinamide, all tested negative. " : Between January 1, 2007 and December 31, 2010. 
resistant to some first-line drugs were given a regimen only suitable for cases with pan-susceptible TB while waiting for full DST results (6-7 weeks for culture and 3-4 weeks for MGIT). The data shown in table 3 are consistent with this possibility as, for example, one third of new cases with resistance to three drugs were subsequently diagnosed with MDR-TB during treatment. Our finding, that the risk of MDR-TB diagnosis during treatment increased linearly with the number of drugs to which the TB strain was resistant at baseline, should be noted for its clinical relevance and is consistent with studies that found poorer outcomes with increased levels of baseline resistance $[16,34]$. Although DOTS is used, we had no information on treatment adherence in these data and thus poor treatment adherence may have contributed to the acquisition of resistance.

In contrast with WHO recommendations [21], Moldovan policy mandates that all TB patients are hospitalised for the intensive phase of therapy. A recent study in Moldova found that in 2009 to 2011, 81\% of all TB patients were hospitalised during treatment with an average length of stay of 92 days [22], coinciding with the time by which half of MDR-TB diagnoses were made in our study. Infection control and isolation practices in Moldova afford opportunities for nosocomial transmission. Face masks are infrequently worn by healthcare providers and patients, MDR-TB cases are only separated from non-MDRTB cases after DST results have become available and all non-MDR-TB patients are grouped together regardless of the presence of resistance to first-line drugs (other than MDR). Due to the high hospitalisation rates in Moldova, it was not possible to assess hospitalisation in our patient-level risk-factor analysis. However, nosocomial transmission is a known source of MDR-TB infection during treatment [12] and the only other study to examine risk factors for MDR-TB appearance during treatment in the FSU identified hospitalisation as the only such risk factor [24]. Infection control was also found to be below internationally recommended standards in some MDR-TB reference centres in Europe [35]. In addition, a study in Moldova that used genotyping to compare baseline and follow-up strains, from 24 individuals in whom MDR-TB appeared during treatment, found that reinfection/mixed infection was responsible for at least half of these events [11]. It is likely that hospitalisation policies in Moldova and other parts of the FSU are contributing to the severity of the current MDR-TB epidemic. Studies have previously indicated that costeffectiveness can be improved by using an ambulatory treatment model [21]; further cost-effectiveness studies may help guide treatment policy in the FSU.

The lack of molecular epidemiological data prohibits any estimate of the relative contributions of the three potential mechanisms for MDR-TB diagnosis during treatment (acquisition of resistance, re-infection and initial mixed infections). We believe that all three mechanisms are likely to play some sort of a role. While there are no published data on the prevalence of the Beijing genotype in Moldova, its high prevalence and association with MDR-TB have been documented in many FSU countries [36, 37], and it has been associated with both an enhanced transmission ability [12] and increased probability of acquiring mutations associated with drug resistance [38]. The prevalence of mixed-strain TB infections, especially mixed drug-resistant and drug-susceptible, in Moldova is currently unknown. Due to challenges with detecting mixed infections in TB [14], few studies have attempted to estimate this prevalence, but these

TABLE 4 Time at which the first follow-up sputum collection occurred among tuberculosis (TB) cases without multidrugresistant (MDR) TB and the percentage that were positive for MDR-TB

Time after initial diagnosis days

New cases

\section{Cases providing first follow-up Positive for MDR-TB"} sputum sample
Previously treated cases

Cases providing first follow-up sputum sample

\begin{tabular}{|c|c|c|c|c|}
\hline$<30$ & $45(2.5)$ & $0(0.0)$ & $22(4.7)$ & $3(13.6)$ \\
\hline $30-59$ & $638(35.8)$ & $33(5.2)$ & 76 (16.4) & $5(6.5)$ \\
\hline $60-89$ & $687(38.6)$ & $36(5.2)$ & $139(30.0)$ & $23(16.5)$ \\
\hline $90-119$ & $127(7.1)$ & 7 (5.5) & $104(22.4)$ & 19 (18.3) \\
\hline $120-149$ & $99(5.6)$ & $7(7.1)$ & $39(8.4)$ & 4 (10.3) \\
\hline $150-179$ & 59 (3.3) & $3(5.1)$ & $25(5.4)$ & $3(12.0)$ \\
\hline $180-209$ & $42(2.4)$ & $5(11.9)$ & $14(3.0)$ & $4(28.6)$ \\
\hline $210-239$ & $26(1.5)$ & $2(7.7)$ & $16(3.4)$ & 5 (31.3) \\
\hline $240-269$ & $20(1.1)$ & $2(10.0)$ & $10(2.2)$ & $3(30.0)$ \\
\hline $270-299$ & $15(0.8)$ & $3(20.0)$ & $10(2.2)$ & $2(20.0)$ \\
\hline $300-329$ & $10(0.6)$ & $3(30.0)$ & $4(0.9)$ & $1(25.0)$ \\
\hline $330-365$ & $12(0.7)$ & $3(25.0)$ & $5(1.1)$ & $2(50.0)$ \\
\hline
\end{tabular}

Data are presented as $\mathrm{n}(\%) .{ }^{\#}$ : percentage of total number; ${ }^{\natural}$ : percentage of those tested. 
types of mixed infections are often undetected [39] and may exacerbate acquisition of resistance [40]. We strongly recommend that molecular epidemiological studies are carried out in Moldova to understand the relative importance of these mechanisms.

Potential limitations of our study include those commonly found when using routinely collected surveillance data. For example, the database that we used only records up to four follow-up cultures per patient, so if any patient had more than four follow-up cultures these would not have been included in our analysis. In addition, despite policy that mandates obtaining and testing sputum at specific time points during treatment, $18 \%$ of patients in this study did not have their first follow-up sputum sample taken until between 4 and 12 months after treatment initiation (table 4). This limits our ability to identify the precise timing of MDR-TB appearance although the direction of error would be towards over-estimating the time of diagnosis and hence MDR-TB appearance may be occurring even more rapidly than estimated.

Good adherence to treatment and access to quality drugs have previously been established as essential components of TB treatment. In our study, the rapid appearance of MDR-TB demonstrates the importance of appropriate treatment and control measures particularly in the early weeks after initial TB diagnosis. This suggests two potential policy changes in Moldova. First, the increased use of rapid drug susceptibility tests would ensure that patients receive appropriate treatment more quickly [41]. While roll-out of these tests has begun since the end of our study period, these results demonstrate the critical need for tests for first-line resistance to prevent early acquisition of drug resistance during therapy. Secondly, these results underscore the need for shifting care from hospitals to ambulatory settings, in accordance with WHO guidelines [19], to reduce the risk of nosocomial transmission. Given the shared epidemiological characteristics of Moldova and other countries in the FSU, these results may reflect a common mechanism exacerbating MDR-TB throughout the entire region.

\section{Acknowledgements}

We thank all those involved in surveillance, laboratory testing and treatment for tuberculosis in the Republic of Moldova.

\section{References}

World Health Organization. Global Tuberculosis Report 2013. Geneva, WHO Press, 2013.

Zignol M, van Gemert W, Falzon D, et al. Surveillance of anti-tuberculosis drug resistance in the world: an updated analysis, 2007-2010. Bull World Health Organ 2012; 90: 111D-119D.

3 Skrahina A, Hurevich $\mathrm{H}$, Zalutskaya A, et al. Alarming levels of drug-resistant tuberculosis in Belarus: results of a survey in Minsk. Eur Respir J 2012; 39: 1425-1431.

4 World Health Organization. Towards Universal Access to Diagnosis and Treatment of Multidrug-Resistant and Extensively Drug-Resistant Tuberculosis by 2015: WHO Progress Report 2011. Geneva, WHO Press, 2011.

5 World Health Organization. Roadmap to Prevent and Combat Drug-Resistant Tuberculosis. Copenhagen, WHO Regional Office for Europe, 2011.

6 National Bureau of Statistics of the Republic of Moldova. Population Census Results Chisinau 2004. www.statistica. md/pageview.php?l=en\&idc=295 Date last accessed: February 6, 2014. Date last updated: February 6, 2014.

7 Jenkins HE, Plesca V, Ciobanu A, et al. Assessing spatial heterogeneity of multidrug-resistant tuberculosis in a highburden country. Eur Respir J 2013; 42: 1291-1301.

8 Soltan V, Henry AK, Crudu V, et al. Increasing tuberculosis case detection: lessons from the Republic of Moldova. Bull World Health Organ 2008; 86: 71-76.

9 Jenkins HE, Ciobanu A, Plesca V, et al. Risk factors and timing of default from treatment for non-multidrugresistant tuberculosis in Moldova. Int J Tuberc Lung Dis 2013; 17: 373-380.

10 Mitchison DA. How drug resistance emerges as a result of poor compliance during short course chemotherapy for tuberculosis. Int J Tuberc Lung Dis 1998; 2: 10-15.

11 Crudu V R-GS, Niemann S, Moraru N, et al. Nosocomial transmission of multidrug-resistant M.tuberculosis strains in a high incidence setting. Int J Tuberc Lung Dis 2010; Suppl. 1, S353.

12 Nodieva A, Jansone I, Broka L, et al. Recent nosocomial transmission and genotypes of multidrug-resistant Mycobacterium tuberculosis. Int J Tuberc Lung Dis 2010; 14: 427-433.

13 van Rie A, Victor TC, Richardson M, et al. Reinfection and mixed infection cause changing Mycobacterium tuberculosis drug-resistance patterns. Am J Respir Crit Care Med 2005; 172: 636-642.

14 Cohen T, van Helden PD, Wilson D, et al. Mixed-strain Mycobacterium tuberculosis infections and the implications for tuberculosis treatment and control. Clin Microbiol Rev 2012; 25: 708-719.

15 Hingley-Wilson SM, Casey R, Connell D, et al. Undetected multidrug-resistant tuberculosis amplified by first-line therapy in mixed infection. Emerg Infect Dis 2013; 19: 1138-1141.

16 Falzon D, Gandhi N, Migliori GB, et al. Resistance to fluoroquinolones and second-line injectable drugs: impact on multidrug-resistant TB outcomes. Eur Respir J 2013; 42: 156-168.

17 World Health Organization. Multidrug and Extensively Drug-Resistant (M/XDR-TB). 2010 Global Report on Surveillance and Response. Geneva, WHO Press, 2010.

18 Crudu V, Soltan V, Burinschi V, et al. Anti-tuberculosis drug resistance surveillance, Republic of Moldova, 2006. Chisinau, Elena-V.I., 2009.

19 World Health Organization. Treatment of Tuberculosis Guidelines, 4th Edn. Geneva, WHO Press, 2010.

20 Ministry of Health and Social Protection, Moldovan Government. $\mathrm{Cu}$ privire la implementarea progamului national de control si profilaxie a tuberculozei in Republica Moldova, pentru anii 2006-2010 [Implementation of 
the National Program for the Control and Prevention of Tuberculosis in Moldova, for 2006-2010.]. Order 180, Annex 11. Chisinau, Ministry of Health and Social Protection, 2006.

21 Falzon D, Jaramillo E, Schunemann HJ, et al. WHO guidelines for the programmatic management of drug-resistant tuberculosis: 2011 update. Eur Respir J 2011; 38: 516-528.

22 Cercone J. Technical Assistance to the Ministry of Health and the National TB Program in Investment Planning and Improvement of TB service Delivery System in The Republic of Moldova: Consultancy Report. Miami, Sanigest, 2013.

23 Turnbull BW. Empirical distribution function with arbitrarily grouped, censored and truncated data. J R Stat Soc Series B Stat Methodol 1976; 38: 290-295.

24 Collett D. Modelling Survival Data in Medical Research. 2nd Edn. Boca Raton, Chapman and Hall/CRC, 2003.

25 SAS Institute Inc. Chapter 48. In SAS/STAT 9.2 User's Guide. The LIFEREG Procedure. Cary, NC, SAS Institute Inc, 2009. pp. 2990-3095.

26 Gelmanova IY, Keshavjee S, Golubchikova VT, et al. Barriers to successful tuberculosis treatment in Tomsk, Russian Federation: non-adherence, default and the acquisition of multidrug resistance. Bull World Health Organ 2007; 85: 703-711.

27 Migliori GB, Zellweger JP, Abubakar I, et al. European Union standards for tuberculosis care. Eur Respir J 2012; 39: 807-819.

28 Shin SS, Keshavjee S, Gelmanova IY, et al. Development of extensively drug-resistant tuberculosis during multidrug-resistant tuberculosis treatment. Am J Respir Crit Care Med 2010; 182: 426-432.

29 Porco TC, Oh P, Flood JM. Antituberculosis drug resistance acquired during treatment: an analysis of cases reported in California, 1994-2006. Clin Infect Dis 2013; 56: 761-769.

30 Ershova JV, Kurbatova EV, Moonan PK, et al. Acquired resistance to second-line drugs among persons with tuberculosis in the United States. Clin Infect Dis 2012; 55: 1600-1607.

31 Lew W, Pai M, Oxlade O, et al. Initial drug resistance and tuberculosis treatment outcomes: systematic review and meta-analysis. Ann Intern Med 2008; 149: 123-34.

32 Cox HS, Niemann S, Ismailov G, et al. Risk of acquired drug resistance during short-course directly observed treatment of tuberculosis in an area with high levels of drug resistance. Clin Infect Dis 2007; 44: 1421-1427.

33 Seung KJ, Gelmanova IE, Peremitin GG, et al. The effect of initial drug resistance on treatment response and acquired drug resistance during standardized short-course chemotherapy for tuberculosis. Clin Infect Dis 2004; 39: $1321-1328$.

34 Migliori GB, Sotgiu G, Gandhi NR, et al. Drug resistance beyond extensively drug-resistant tuberculosis: individual patient data meta-analysis. Eur Respir J 2013; 42: 169-179.

35 Sotgiu G, D'Ambrosio L, Centis R, et al. TB and M/XDR-TB infection control in European TB reference centres: the Achilles' heel? Eur Respir J 2011; 38: 1221-1223.

36 Cox HS, Kubica T, Doshetov D, et al. The Beijing genotype and drug resistant tuberculosis in the Aral Sea region of Central Asia. Respir Res 2005; 6: 134.

37 Niemann S, Diel R, Khechinashvili G, et al. Mycobacterium tuberculosis Beijing lineage favors the spread of multidrug-resistant tuberculosis in the Republic of Georgia. J Clin Microbiol 2010; 48: 3544-3550.

38 Ford CB, Shah RR, Maeda MK, et al. Mycobacterium tuberculosis mutation rate estimates from different lineages predict substantial differences in the emergence of drug-resistant tuberculosis. Nat Genet 2013; 45: 784-790.

39 Köser CU, Bryant JM, Becq J, et al. Whole-genome sequencing for rapid susceptibility testing of M. tuberculosis. N Engl J Med 2013; 369: 290-292.

40 Sun G, Luo T, Yang C, et al. Dynamic population changes in Mycobacterium tuberculosis during acquisition and fixation of drug resistance in patients. J Infect Dis 2012; 206: 1724-1733.

41 Weyer K, Mirzayev F, Migliori GB, et al. Rapid molecular TB diagnosis: evidence, policy making and global implementation of Xpert MTB/RIF. Eur Respir J 2013; 42: 252-271. 